Can. J. Math., Vol. XXIX, No. 2, 1977, pp. 392-399

\title{
ON TRANSFORMATION AND OSCILLATION OF LINEAR DIFFERENTIAL SYSTEMS
}

\author{
DONALD F. ST. MARY
}

1. Introduction. In this paper we study second order linear differential systems. We examine the relationship between oscillation of $n$-dimensional systems and certain associated $m$-dimensional systems, where $m \leqq n$. Several theorems are presented which unify and encompass in the linear case a number of results from the literature. In particular, we present a transformation which extends an oscillation theorem due to Allegretto and Erbe [1], and a comparison theorem due to Kreith [9], and explains some work of Howard [7].

We shall be concerned with the differential system

$$
l[u] \equiv\left[R(t) u^{\prime}+Q(t) u\right]^{\prime}-\left[Q^{*}(t) u^{\prime}-P(t) u\right]=0,
$$

where each of the $n \times n$ matrix functions $R(t), P(t), Q(t)$ has complex valued entries which are continuous on a given subinterval $I$ of the real line and $R(t)$ and $P(t)$ are hermitian on $I$. A solution of $(1.1)$ on $I$ is an $n$-dimensional vector function $u(t)$, for which $u(t)$, and $v(t)=R(t) u^{\prime}(t)+Q(t) u(t)$ are continuously differentiable on $I$, and (1.1) holds on $I$.

Definition. The system $(1.1)$ is said to be oscillatory on the interval $[a, \infty)$ if for each $\alpha \geqq a$ there is a $\beta>\alpha$ and a solution $u$ of $(1.1)$ defined on $[a, \infty)$ such that $u(\alpha)=0, v(\alpha) \neq 0$ and $u(\beta)=0$.

Corresponding to the vector system (1.1), we have the matrix differential system

$$
(1.1 \mathrm{~m}) \quad l[U] \equiv\left[R(t) U^{\prime}+Q(t) U\right]^{\prime}-\left[Q^{*} U^{\prime}-P(t) U\right]=0,
$$

with a solution, an $n \times s$ matrix, $U(t), V(t)=R(t) U^{\prime}(t)+Q(t) U(t)$, defined in a manner analogous to the earlier definition, $1 \leqq s \leqq n$. The relationship between $(1.1)$ and $(1.1 \mathrm{~m})$ is that $U$ is a solution of $(1.1 \mathrm{~m})$ if and only if $u(t)=U(t) \gamma$ is a solution of (1.1) for all $s$-vectors $\gamma$.

From time to time we shall require $R(t)$ to be nonsingular and/or positive definite $R>0$, but we wish to remark that at the present time no such assumptions are being made, also we do not make a general assumption of positiveness on $P(t)$.

2. Oscillation. In this section we present an oscillation theorem which we will show is the foundation of a number of recent results in the literature. First, we shall have need of some preliminary definitions and theorems.

Received May 26, 1976 and in revised form, October 15, 1976. 
Two solutions $u_{1}(t), u_{2}(t)$ of $(1.1)$ on $I$ are said to be isotropic [3, Chapter 2] or (mutually) conjoined [11, Chapter 7], if $v_{2}^{*}(t) u_{1}(t)-u_{2}^{*}(t) v_{1}(t)$, which is constant on $I$, has the value zero. Let $U(t)$ be an $n \times n$ solution of $(1.1 \mathrm{~m})$ on $I$, if the column vectors of $U(t)$ are mutually conjoined, i.e., $V^{*} U-U^{*} V=0$ on $I$, and the column vectors of the $2 n \times n$ matrix $(U(t) ; V(t))$, (the first $n$ rows is $U$, the next $n$ rows is $V$ ), are linearly independent on $I$ then $U(t)$ is called a conjoined basis for $(1.1 \mathrm{~m})$.

For a given compact interval $[a, b]$ let $D[a, b]=D_{n}[a, b]$ denote the set of all $n$-vector functions $\eta$, which are absolutely continuous on $[a, b]$ and for which $R \eta^{\prime}+Q \eta$ is Lebesgue square integrable on $[a, b]$. The subclass of $D[a, b]$ on which $\eta(a)=\eta(b)=0$ will be denoted by $D_{0}[a, b]$. For $\eta \in D[a, b]$ we shall denote by $J[\eta ; a, b]$ the functional

$$
J[\eta ; a, b]=\int_{a}^{b}\left\{\eta^{* \prime}\left[R \eta^{\prime}+Q \eta\right]+\eta^{*}\left[Q^{*} \eta^{\prime}-P \eta\right]\right\} d t .
$$

We remark that an $n \times s$ matrix, $1 \leqq s \leqq n$, may be inserted in this functional in place of $\eta$. The following is a standard identity involving $J[\eta ; a, b]$ and follows from an integration by parts.

Lemma 2.1. If $\eta \in D[a, b]$ and $R \eta^{\prime}+Q \eta$ is absolutely continuous on $[a, b]$, then

$$
J[\eta ; a, b]=\left.\eta^{*}\left(R \eta^{\prime}+Q \eta\right)\right|_{a} ^{b}-\int_{a}^{b} \eta^{*} l[\eta] d t .
$$

In particular, if $l[\eta]=0$ and $\eta(a)=\eta(b)=0$ then $J[\eta ; a, b]=0$.

We shall show that recent results on oscillation are based on the following theorem.

Theorem 2.2. Let $R(t)>0$ on $[a, \infty)$. Then $(1.1)$ is oscillatory on $[a, \infty)$ if and only if there exists a sequence of intervals $\left[\alpha_{k}, \beta_{k}\right], a \leqq \alpha_{k}<\beta_{k}$, with $\alpha_{k} \rightarrow \infty$ with $k$, and nontrivial functions $\eta_{k}$ in $D_{0}\left[\alpha_{k}, \beta_{k}\right]$ such that $J\left[\eta_{k} ; \alpha_{k}, \beta_{k}\right] \leqq 0$.

Proof. It is well-known that $J[\eta ; a, b]$ is positive definite on $D_{0}[a, b]$ if and only if there exists a conjoined basis $U(t)$ which is nonsingular on $[a, b]$. Assume the conditions hold and (1.1) is not oscillatory then for some $\alpha \geqq a$, the conjoined basis $U(t)$ satisfying $U(\alpha)=0, V(\alpha)=E_{n}$ (the $n \times n$ identity matrix) is nonsingular for all $t>\alpha$. Let $\alpha_{k}>\alpha$ then $J\left[\eta ; \alpha_{k}, \beta_{k}\right]$ is positive definite on $D_{0}\left[\alpha_{k}, \beta_{k}\right]$, contradicting the existence of $\eta_{k}$. The converse is immediate in view of Lemma 2.1.

3. Transformation and oscillation. In this section we shall present a transformation of (1.1) and several theorems on oscillation.

Theorem 3.1. Let $H(t)$ be an $n \times r$ matrix function, $1 \leqq r \leqq n$, such that 
$H$ and $R H^{\prime}+Q H$ are absolutely continuous on I and

$$
\left(R H^{\prime}+Q H\right)^{*} H=H^{*}\left(R H^{\prime}+Q H\right) \text {, on } I
$$

and put $\mathscr{R}[t]=H^{*} R H, \mathscr{P}(t)=H^{*}[[H]$. If

$$
\mathscr{L}[X]=\left(\mathscr{R}(t) X^{\prime}\right)^{\prime}+\mathscr{P}(t) X
$$

for $X$ an $r \times s$ matrix function, $1 \leqq s \leqq r$, with $X$ and $R H X^{\prime}$ absolutely continuous on $I$, then

$$
H^{*}[[U]=\mathscr{L}[X]
$$

where $U=H X$.

Furthermore,

$$
J[U ; a, b]=\left.(H X)^{*}\left(R H^{\prime}+Q H\right) X\right|_{a} ^{b}+\int_{a}^{b}\left\{X^{*} \mathscr{R} X^{\prime}-X^{*} \mathscr{P} X\right\} d t .
$$

Proof. $\mathscr{L}[X]=H^{* \prime}\left(R H X^{\prime}\right)+H^{*}\left(R H X^{\prime}\right)^{\prime}$

$$
\begin{aligned}
& \quad+H^{*}\left(R H^{\prime}+Q H\right)^{\prime} X-H^{*}\left(Q^{*} H^{\prime}-P H\right) X \\
& =H^{*}\left[\left(R H^{\prime}+Q H\right)^{\prime} X+\left(R H^{\prime}+Q H\right) X^{\prime}+\left(R H X^{\prime}\right)^{\prime}\right] \\
& \quad-H^{*}\left(R H^{\prime}+Q H\right) X^{\prime}+H^{* \prime} R H X^{\prime}-H^{*}\left(Q^{*} H^{\prime}-P H\right) X \\
& =H^{*}\left[\left(R H^{\prime}+Q H\right) X+R H X^{\prime}\right]^{\prime}-(R H+Q H)^{*} H X^{\prime} \\
& \quad+H^{* \prime} R H X^{\prime}-H^{*}\left(Q^{*} H^{\prime}-P H\right) X \\
& =H^{*} l[U] .
\end{aligned}
$$

It follows from Lemma 2.1 and (3.3) that

$$
J[U ; a, b]=\left.(H X)^{*}\left[R(H X)^{\prime}+Q(H X)\right]\right|_{a} ^{b}-\int_{a}^{b} X^{*} \mathscr{L}[X] d t .
$$

Now an integration by parts of the last integral yields the desired conclusion.

The following two corollaries follow from the theorem in a straightforward manner using the definitions given earlier.

Corollary 3.2. If $H(t)$ is an $n \times n$ nonsingular matrix satisfying the hypothesis of the theorem, then $X$ is a conjoined basis for $\mathscr{L}[X]=0$ on I if and only if $U=H X$ is a conjoined basis for $(1.1 \mathrm{~m})$ on $I$.

Corollary 3.3. If $H(t)$ is an $n \times n$ nonsingular matrix satisfying the hypothesis of the theorem, then the system (1.1) is oscillatory on $[a, \infty)$ if and only if the system $\mathscr{L}[X]=0$ is oscillatory on $[a, \infty)$.

An interesting application of Corollary 3.3 occurs when, in (1.1), $R \equiv E_{n}$, $Q \equiv 0$ and $\int_{t}^{\infty} P(s) d s \equiv S(t)$ exists (finitely). One takes $H$ to be the fundamental solution of the system $H^{\prime}=2 S(t) H$, then $\mathscr{R}=H^{*} H>0$ and $\mathscr{P}=$ $H^{*}\left[4 S^{2}(t)-P(t)\right] H$. It follows that if $4\left[\int_{i}^{\infty} P(s) d s\right]^{2} \leqq P(t)$ then $\mathscr{L}[X]$ is non- 
oscillatory and hence $U^{\prime \prime}+P(t) U=0$ is nonoscillatory (the result itself is known and goes back to Wintner). The setting of this example is studied in [5].

The next theorem is the kind of result which is of primary interest in this paper. The result obtains oscillation of (1.1) on considering a related (in this case, scalar) equation. Analogous results appear in Simons [13].

For $A(t)$ an $n \times n$ matrix, hermitian and continuous on $[a, \infty)$, we shall let $\lambda_{1}(A(t)), \ldots, \lambda_{n}(A(t))$ be the characteristic values of $A(t)$, with the notation so chosen that $\lambda_{1}(A(t)) \leqq \ldots \leqq \lambda_{n}(A(t))$ for each $t$ in $[a, \infty)$. The functions $\lambda_{i}(A(t))$ are continuous.

THEOREM 3.4. Let $R>0$, and $q_{i i}$ be real valued and continuously differentiable for each $i, Q=\left(q_{i j}\right)$. If the scalar differential equation

$$
\left((\operatorname{tr}(R(t))) y^{\prime}\right)^{\prime}+\left(\operatorname{tr}\left(Q^{\prime}(t)+P(t)\right)\right) y=0
$$

is oscillatory on $[a, \infty)$, then $(1.1)$ is oscillatory on $[a, \infty)$. In particular, if $\left(\lambda_{n}(R(t)) y^{\prime}\right)^{\prime}+\lambda_{1}\left(Q^{\prime}(t)+P(t)\right) y=0$ is oscillatory, then (1.1) is oscillatory.

Proof. If we denote the $J$-functional associated with $(3.5)$ by $J_{s}[\eta ; a, b]$, then Theorem 2.2 implies that there exists a sequence of intervals $\left[\alpha_{k}, \beta_{k}\right]$ with $\alpha_{k} \rightarrow \infty$ and nontrivial scalar functions $\eta_{k}$ in $D_{0}\left[\alpha_{k}, \beta_{k}\right]$ such that $J_{s}\left[\eta_{k} ; \alpha_{k}, \beta_{k}\right] \leqq$ 0 . In Theorem 3.1 take $H$ to be the $n$-vector all of whose components are zero except the $i$-th which is 1 , and take $X$ to be $\eta_{k}$, then for $\nu_{i}=H \eta_{k}$, it follows from (3.4) that

$$
J\left[\nu_{i} ; \alpha_{k}, \beta_{k}\right]=\int_{\alpha_{k}}^{\beta_{k}}\left\{\eta_{k}{ }^{\prime} r_{i i} \eta_{k}{ }^{\prime}-\eta_{k}\left(q_{i i}{ }^{\prime}+p_{i i}\right) \eta_{k}\right\} d t,
$$

here $R(t)=\left(r_{i j}(t)\right)$, etc. If one sums on $i$ in (3.6) the right hand side becomes $J_{s}\left[\eta_{k} ; \alpha_{k}, \beta_{k}\right]$, which is less than or equal to zero, thus for some $i, J\left[\nu_{i} ; \alpha_{k}, \beta_{k}\right] \leqq 0$. The first statement now follows from Theorem 2.2.

The second statement follows from the Sturm Comparison Theorem and the fact that for a hermitian matrix $A(t), n \lambda_{n}(A) \geqq \operatorname{tr}(A)$, and $n \lambda_{1}(A) \leqq \operatorname{tr}(A)$.

The following corollary utilizes scalar criteria due to Hartman (see, e.g., [6, XI, Theorem 7.3]). Eliason [4] obtains a related theorem using entirely different methods.

COROLlary 3.5. Let $Q \equiv 0, R \equiv E_{n}$. If

$$
\lambda_{n}\left[\frac{1}{T} \int^{T}\left(\int^{t} P(s) d s\right) d t\right] \rightarrow+\infty \quad \text { as } T \rightarrow+\infty
$$

and

$$
\lim \inf \lambda_{1}\left[\frac{1}{T} \int^{T}\left(\int^{t} P(s) d s\right) d t\right]>-\infty \quad \text { as } T \rightarrow+\infty
$$

then (1.1) is oscillatory. In particular if $P \geqq 0$ and $\int^{\infty}\|P\| d s=+\infty$ then (1.1) is oscillatory. 
The corollary follows from the theorem and Hartman's theorem since the hypothesis insures that

$$
\frac{1}{T} \int^{T}\left(\int^{t} \frac{\operatorname{tr}(P(s))}{n} d s\right) d t \rightarrow+\infty \quad \text { as } T \rightarrow+\infty
$$

and hence (3.5) is oscillatory.

We remark that the second statement of the corollary is not true if the nonnegative definiteness of $P$ is dropped. To see this choose $P(t)=\operatorname{diag}$ $(\sin t / 4 t,-\sin t / 4 t)$ then $\|P(t)\|=|\sin t| / 4 t$. The scalar equations $u^{\prime \prime} \pm$ $(\sin t / 4 t) u=0$ are nonoscillatory [15] and thus the (diagonal) system (1.1) is nonoscillatory.

The following theorem extends the result of Allegretto and Erbe [1] , and others, in the case under consideration here.

Theorem 3.6. Let $R(t)>0$ on $[a, \infty)$, and let $H(t)$ be an $n \times r$ matrix function, $1 \leqq r \leqq n$, such that $H$ and $R H^{\prime}+Q H$ are absolutely continuous on $[a, \infty)$ and (3.1) holds on $[a, \infty)$. If the system

$$
\mathscr{L}[x]=\left(\mathscr{R} x^{\prime}\right)^{\prime}+\mathscr{P} x=0,
$$

$\mathscr{R}=H^{*} R H, \mathscr{P}=H^{*} l[H]$, is oscillatory on $[a, \infty)$, then the system (1.1) is oscillatory on $[a, \infty)$.

Proof. Let $\alpha \geqq a$. Since $\mathscr{L}[x]=0$ is oscillatory there is a $\beta>\alpha$ and an $r$-dimensional vector solution $x(t)$ of $\mathscr{L}[x]=0$ such that $x(\alpha)=0, x(\beta)=0$ and $\mathscr{R}(\alpha) x^{\prime}(\alpha) \neq 0$. Now take $X(t)$ of Theorem 3.1 to be $x(t)$ and put $\eta(t)=$ $H x$, then $\eta(\alpha)=\eta(\beta)=0$, and (3.4) plus Lemma 2.1 yield

$$
J[\eta ; \alpha, \beta]=\int_{\alpha}^{\beta}\left\{x^{* \prime} \mathscr{R} x-x^{*} \mathscr{P} x\right\} d t=0 .
$$

Furthermore, $\eta(t) \not \equiv 0$, for if so, then $0 \equiv \eta^{\prime}(t)=H^{\prime} x+H x^{\prime}$ and thus $\mathscr{R}(\alpha) x^{\prime}(\alpha)=\left(H^{*} R H x^{\prime}\right)(\alpha)=-\left(H^{*} R H^{\prime} x\right)(\alpha)=0$, a contradiction. The conclusion of the theorem now follows from Theorem 2.2 .

Allegretto and Erbe have shown that a number of interesting results follow from theorems such as the previous one. The corollary below shows that to determine oscillation of (1.1) one may consider certain subsystems. If a one dimensional such subsystem is used one obtains the result first proved by Swanson [18] (see also Barrett [2]), that if a "diagonal equation" oscillates then the original system is oscillatory. It follows that all scalar oscillation criteria yield, in a trivial manner, oscillation criteria for systems (1.1), for example, one can take $H$ to be an $n \times 1$ vector and the resulting transformed system is scalar.

For a given $n \times n$ matrix $A=\left(a_{i j}\right)$, let $A_{\gamma}$ be the $r \times r$ submatrix of $A$, $1 \leqq r \leqq n$ obtained by deleting each row and column of $A$ except row and 
column $i_{1}, i_{2}, \ldots, i_{r}$, where $1 \leqq i_{1} \leqq \ldots \leqq i_{r} \leqq n$, here $\gamma=\left(i_{1}, \ldots, i_{r}\right)$. If one chooses $H_{(\gamma)}$ to be the $n \times r$ submatrix of the $n \times n$ identity matrix $E$, obtained by deleting each column of $E$ except column $i_{1}, \ldots, i_{r}$, then $A_{\gamma}=$ $H_{(\gamma)} * A H_{(\gamma)}$.

Corollary 3.7. Let $R>0$ and $Q$ be continuously differentiable. If for some $\gamma$, $Q_{\gamma}{ }^{*}=Q_{\gamma}$, and the system

$$
\left(R_{\gamma}(t) u^{\prime}\right)^{\prime}+\left(Q_{\gamma}{ }^{\prime}(t)+P_{\gamma}(t)\right) u=0
$$

is oscillatory, then (1.1) is oscillatory.

This statement follows directly from the theorem on choosing $H$ to be $H_{(\gamma)}$.

In [7], H. C. Howard discusses oscillation of systems of the form (1.1) with $Q \equiv 0$, by introducing and placing conditions on new functions which are related to $R$ and $P$. Theorem 3.1 makes it possible to understand the relationship between these new functions and the original $R$ and $P$. If one takes $H(t)=$ $\sqrt{g(t) E}$, here $g(t)$ is a positive scalar function for which $g$, and $R g^{\prime}$ are $C^{1}$, then Howard's functions are obtained as the coefficients of the system (3.2), in particular,

$$
\begin{aligned}
& \mathscr{R}(t)=g R(t) \\
& \mathscr{P}(t)=g P(t)-(1 / 4) R(t)\left(g^{\prime}\right)^{2} g^{-1}+(1 / 2)\left(R(t) g^{\prime}\right)^{\prime} .
\end{aligned}
$$

For $g(t)=t$, one obtains the Kneser-type conditions, see, e.g., [11].

With the help of the Fite-Wintner-Leighton theorem [10], the following theorem encompasses, in the linear case, Howard's original theorem, its extension by Kartsatos [8], and the Noussair and Swanson theorem.

Theorem 3.8. Let $R>0$ and continuously differentiable, $Q \equiv 0$. If for some $\gamma$, the scalar equation

$$
\left(\left(\operatorname{tr}\left(\mathscr{R}_{\gamma}(t)\right)\right) y^{\prime}\right)^{\prime}+\left(\operatorname{tr}\left(\mathscr{P}_{\gamma}(t)\right)\right) y=0
$$

$\mathscr{R}$ and $\mathscr{P}$ as in (3.7), is oscillatory, then (1.1) is oscillatory.

Proof. By Theorem 3.4

$$
\left(\mathscr{R}_{\gamma} y^{\prime}\right)^{\prime}+\mathscr{P}_{\gamma} y=0
$$

is oscillatory and thus by Corollary 3.7, (1.1) is oscillatory.

We wish to remark that a natural choice for $H(t)$, when $R(t)>0$, is the inverse of the positive definite square root of $R(t)$, for then $\mathscr{R}(t)$ becomes the identity matrix.

4. Comparison and oscillation. In the previous section many of the results are obtained via an indirect comparison of two systems. In this section we present a more direct comparison theorem which utilizes the $H$-transformation. 
Consider two systems of the form (1.1),

$$
l_{i}[u]=\left[R_{i}(t) u^{\prime}+Q_{i}(t) u\right]^{\prime}-\left[Q_{i}^{*}(t) u^{\prime}-P_{i}(t) u\right]=0, \quad i=1,2,
$$

where the conditions on $R_{i}, Q_{i}$, and $P_{i}$ are the same as those on (1.1).

The following result is a significant extension of the theorem in Kreith [9] for the case under consideration here and encompasses the standard Sturm type comparison theorem.

TheOREM 4.1. Let $R_{2}>0$ on $[a, \infty)$. Let $H(t)$ be an $n \times r$ matrix function, $1 \leqq r \leqq n$, such that $H$ and $R_{i} H^{\prime}+Q_{i} H$ are absolutely continuous on $[a, \infty)$, $\left(R_{i} H^{\prime}+Q_{i} H\right)^{*} H=H^{*}\left(R_{i} H^{\prime}+Q_{i} H\right)$, and $\mathscr{R}_{i}(t)=H^{*} R_{i} H, \mathscr{P}_{i}(t)=H^{*} l_{i}[H]$ and $\mathscr{L}_{i}[X]=\left(\mathscr{R}_{i} X^{\prime}\right)^{\prime}+\mathscr{P}_{i} X$ for $i=1,2$. If $\mathscr{L}_{1}[x]=0$ is oscillatory on $[a, \infty)$ and if there exists a sequence of real numbers $\left(\alpha_{k}\right), \alpha_{k} \rightarrow \infty$ such that for each $k=1,2, \ldots$

$$
\int_{\alpha_{k}}^{\tau}\left\{\eta^{* \prime} H^{*}\left(R_{1}-R_{2}\right) H \eta^{\prime}+\eta^{*} H^{*}\left(l_{2}-l_{1}\right)[H] \eta\right\} d t
$$

is nonnegative for all $\eta \in D_{0}\left[\alpha_{k}, \tau\right] \subseteq D_{r}[a, b], \tau>\alpha_{k}$, then $l_{2}[u]=0$ is oscillatory on $[a, \infty)$.

Proof. We shall verify that the condition of Theorem 2.2 is satisfied. For a given $\alpha_{k}, \mathscr{L}_{1}[x]$ oscillatory implies the existence of a solution $x$ of $\mathscr{L}_{1}[x]=0$ and a number $\beta_{k}>\alpha_{k}$ such that $x\left(\alpha_{k}\right)=x\left(\beta_{k}\right)=0,\left(\mathscr{R}_{1} x^{\prime}\right)\left(\alpha_{k}\right) \neq 0$. Now

$$
\begin{aligned}
0 \leqq & \int_{\alpha_{k}}^{\beta_{k}}\left\{x^{* \prime} H^{*}\left(R_{1}-R_{2}\right) H x^{\prime}+x^{*} H^{*}\left(l_{2}-l_{1}\right)[H] x\right\} d t \\
& =\int_{\alpha_{k}}^{\beta_{k}}\left\{x^{* \prime} \mathscr{R}_{1} x^{\prime}-x^{*} \mathscr{P}_{1} x\right\} d t-\int_{\alpha_{k}}^{\beta_{k}}\left\{x^{* \prime} \mathscr{R}_{2} x^{\prime}-x^{*} \mathscr{P}_{2} x\right\} d t \\
& \equiv \mathscr{J}_{1}\left[x ; \alpha_{k}, \beta_{k}\right]-\mathscr{J}_{2}\left[x ; \alpha_{k}, \beta_{k}\right] .
\end{aligned}
$$

But $\mathscr{J}_{1}\left[x ; \alpha_{k}, \beta_{k}\right]=0$ by Lemma 2.1 so $\mathscr{J}_{2}\left[x ; \alpha_{k}, \beta_{k}\right] \leqq 0$ and since, by (3.4), $\mathscr{J}_{2}\left[x ; \alpha_{k}, \beta_{k}\right]=J_{2}\left[H x ; \alpha_{k}, \beta_{k}\right]$, it follows that $J_{2}\left[H x ; \alpha_{k}, \beta_{k}\right] \leqq 0$.

The following corollary is a trivial consequence of the theorem obtained on taking $H$ to be $E$, the $n \times n$ identity matrix, and observing that $R(t)-$ $\lambda_{1}(R(t)) E$ and $\lambda_{n}(P(t)) E-P(t)$ are nonnegative definite matrices.

Corollary 4.2. Let $R(t)>0$ and $Q(t) \equiv 0$. If $(1.1)$ is oscillatory then the scalar equation

$$
\left(\left(\lambda_{1}(R(t))\right) y^{\prime}\right)^{\prime}+\left(\lambda_{n}(P(t))\right) y=0
$$

is oscillatory.

We remark that the converse of this theorem is not true as can be seen from the example following Corollary 3.5. 


\section{REFERENCES}

1. W. Allegretto and L. Erbe, Oscillation criteria for matrix differential inequalities, Can. Math. Bull. 16 (1973), 5-10.

2. J. H. Barrett, Matrix systems of second order differential equations, Portugaliae Mathematica 14 (1955), 79-89.

3. W. A. Coppel, Disconjugacy, Lecture Notes in Mathematics 220 (Springer-Verlag, Berlin, Heidelberg, New York, 1971).

4. S. B. Eliason, Results on oscillation for a second order linear matrix system of differential equations, submitted for publication.

5. S. B. Eliason and D. F. St. Mary, On oscillation of linear differential systems, to appear.

6. P. Hartman, Ordinary differential equations (John Wiley \& Sons, New York, 1964).

7. H. C. Howard, Oscillation criteria for matrix differential equations, Can. J. Math. 19 (1967), 184-199.

8. A. G. Kartsatos, Oscillation of nonlinear systems of matrix differential equations, Proc. Amer. Math. Soc. 30 (1971), 97-101.

9. K. Kreith, Oscillation criteria for nonlinear matrix differential equations, Proc. Amer. Math. Soc. 26 (1970), 270-272.

10. W. Leighton, On self-adjoint differential equations of second order, J. London Math. Soc. 27 (1952), 37-47.

11. E. S. Noussair and C. A. Swanson, Oscillation criteria for differential systems, J. Math. Anal. Appl. 36 (1971), 575-580.

12. W. T. Reid, Ordinary differential equations (John Wiley \& Sons, New York, 1971).

13. W. Simons, Disconjugacy criteria for systems of self-adjoint differential equations, J. London Math. Soc. 6 (1973), 373-381.

14. C. A. Swanson, Oscillation criteria for nonlinear matrix differential inequalities, Proc. Amer. Math. Soc. 24 (1970), 824-827.

15. D. Willet, On the oscillatory behavior of the solutions of second order linear differential equations, Ann. Polon. Math. 21 (1969), 175-194.

University of Massachusetts,

Amherst, Massachusetts;

University of Oklahoma,

Norman, Oklahoma 